\title{
Attention to Features Precedes Attention to Locations in Visual Search: Evidence from Electromagnetic Brain Responses in Humans
}

\author{
Jens-Max Hopf, ${ }^{1,2}$ Kai Boelmans, ${ }^{1}$ Mircea Ariel Schoenfeld, ${ }^{1}$ Steven J. Luck, ${ }^{3}$ and Hans-Jochen Heinze ${ }^{1}$ \\ ${ }^{1}$ Department of Neurology II, Otto-von-Guericke-University, D-39120 Magdeburg, Germany, ${ }^{2}$ Leibniz-Institute for Neurobiology, D-39120 Magdeburg, \\ Germany, and ${ }^{3}$ Department of Psychology, University of Iowa, Iowa City, Iowa 52242-1407
}

\begin{abstract}
Single-unit recordings in macaque extrastriate cortex have shown that attentional selection of nonspatial features can operate in a location-independent manner. Here, we investigated analogous neural correlates at the neural population level in human observers by using simultaneous event-related potential (ERP) and event-related magnetic field (ERMF) recordings. The goals were to determine (1) whether task-relevant features are selected before attention is allocated to the location of the target, and (2) whether this selection reflects the locations of the relevant features. A visual search task was used in which the spatial distribution of nontarget items with attended feature values was varied independently of the location of the target. The presence of task-relevant features in a given location led to a change in ERP/ERMF activity beginning $\sim 140 \mathrm{msec}$ after stimulus onset, with a neural origin in the ventral occipito-temporal cortex. This effect was independent of the location of the actual target. This effect was followed by lateralized activity reflecting the allocation of attention to the location of the target (the well known N2pc component), which began at $\sim 170$ msec poststimulus. Current source localization indicated that the allocation of attention to the location of the target originated in more anterior regions of occipito-temporal cortex anterior than the feature-related effects. These findings suggest that target detection in visual search begins with the detection of task-relevant features, which then allows spatial attention to be allocated to the location of a likely target, which in turn allows the target to be positively identified.
\end{abstract}

Key words: attention; visual; search; featural; spatial; human

\section{Introduction}

Psychophysical and neurophysiological evidence indicates that attention can be focused to spatial locations independent of eye fixations (Posner, 1980; Hillyard and Münte, 1984; Moran and Desimone, 1985; Motter, 1993; Heinze et al., 1994; Brefczynski and DeYoe, 1999). Attention may also be allocated to nonspatial features (Corbetta et al., 1990; O'Craven et al., 1997), and this may occur in a location-independent manner (Motter, 1994; Treue and Martinez Trujillo, 1999). Motter (1994) has shown that the firing rate of color-responsive cells in macaque area V4 is enhanced when the color of a stimulus in the receptive field of the cell matches the color of a previous cue. This effect depended on the presence of the precued color and not on the location of the attended items. Treue and Martinez Trujillo (1999) demonstrated similar effects in macaque middle temporal visual area

Received July 30, 2003; revised Dec. 4, 2003; accepted Dec. 6, 2003.

This work was supported by Bundesministerium für Bildung und Forschung Center for Advanced Imaging Grant 01G000202, Grant He1531/3-5 from the Deutsche Forschungs-gemeinschaft to H.-J.H., and Grants MH56877 and MH63001 from the National Institute of Mental Health to S.J.L. We are grateful to E. Düzel and A. RichardsonKlavehn for comments on this manuscript.

Correspondence should be addressed to Dr. Jens-Max Hopf, Department of Neurology II, Otto-vonGuericke-University of Magdeburg, Leipziger Strasse 44, 39120 Magdeburg, Germany. E-mail: jens-max.hopf@medizin.uni-magdeburg.de.

DOI:10.1523/JNEUROSCI.3564-03.2004

Copyright $\odot 2004$ Society for Neuroscience $\quad 0270-6474 / 04 / 241822-11 \$ 15.00 / 0$
(MT) using bilateral moving dot patterns. Attending to a certain direction of movement in one visual field also enhanced firing of MT cells tuned for that movement direction in the unattended visual field [for related functional magnetic resonance imaging (fMRI) evidence, see Saenz et al., 2002].

Feature-based attention may be important in visual search, because it could provide a map of likely target locations (Motter, 1994). Feature-based location maps of various kinds have been postulated in many theories of visual search (Treisman and Gelade, 1980; Treisman and Sato, 1990; Wolfe, 1994; Cave, 1999), and behavioral studies have demonstrated that attending to features provides access to their spatial locations (Kim and Cave, 1995, 2001; Shui-I and Sperling, 1996). Once relevant features have been located, spatial attention may then be allocated to the locations containing those features (Wolfe et al., 1989; Treisman and Sato, 1990), and this may then allow a suppression of information from the other locations, improving the perceptual analysis at attended locations (Chelazzi et al., 1993; Luck et al., 1997a).

Although this progression from feature- to location-based selection is a common idea in theories of attention, there is little direct evidence for such progression. Most neurophysiological studies have examined only feature-based or only location-based selection (Motter, 1993, 1994; Luck et al., 1997b, Connor et al., 1997), and behavioral studies cannot usually provide direct evi- 
dence about the timing of attentional processes. The present study combines event-related potential (ERP)/event-related magnetic field (ERMF) recordings to test the hypothesis that the spatial distribution of task-relevant features is computed rapidly, followed by the focusing of attention onto the object that is most likely to be the target.

In ERP studies of visual search, the first index of focusing attention onto the location of the target is $\mathrm{N} 2 \mathrm{pc}$, a negative-going deflection in the N2 time range $(180-300 \mathrm{msec})$ that is largest at posterior scalp sites, contralateral to the location of the attended item (Luck and Hillyard, 1994a,b; Luck et al., 1997a). The N2pc seems to be closely related to modulations of single-unit activity in macaque extrastriate/inferotemporal areas (Chelazzi et al., 1993, 2001) and originates from ventral occipito-temporal cortex in humans (Hopf et al., 2000, 2002a). Our main question was whether the N2pc component would be preceded by activity related to the detection and localization of task-relevant features.

\section{Materials and Methods}

Stimuli and procedure (experiment 1). As illustrated in Figure $1 a$, each stimulus array consisted of one cluster of C-shaped items (diameter, $0.85^{\circ}$; gap width, $0.6^{\circ}$ ) in each visual field, centered $2.2^{\circ}$ below and $3.6^{\circ}$ lateral to the fixation point. Each cluster contained a ring of six blue Cs surrounding, at a distance of $1.5^{\circ}$, either a red $\mathrm{C}$ or a green C. A red C was in one cluster, and a green $\mathrm{C}$ was in the other, and the side containing a particular color varied randomly from trial to trial. The gap of each central $\mathrm{C}$ could be on either the left or right side, varying unpredictably independently. At the beginning of each trial block, the subject was instructed that either the red $\mathrm{C}$ or the green $\mathrm{C}$ would be the target for that block (the order alternated across blocks). Subjects were required to report the position of the gap of the target with a two alternative button press of the right hand (left gap $=$ index finger, right gap $=$ middle finger). In this manner, a given stimulus array could serve as a left target array or a right target array, depending on which color was the target for that block. The data were then collapsed across the red target and green target conditions to control for any spatial inhomogeneities attributable to target color. Note that because red and green, but never blue, served as target colors in this experiment, it may be possible that these colors may have gained some significance even when they served as distractors. This may have caused some slight attentional capture toward the nontarget side, an effect that would not be controlled for by collapsing across the red target and green target conditions. However, because this would have affected all four experimental conditions to an equal extent, this would not influence the conclusions that can be drawn from this experiment.

The targets in this experiment had two relevant features, namely a color (which remained constant throughout a trial block) and an orientation (which varied but was always along the horizontal axis). The distractors within a given cluster either shared the same general orientation as the possible target shapes, with a gap on the left or right side, or did not share this orientation, with a gap on the top or bottom. We refer to distractors with the same general orientation as the target category as relevant-orientation distractors (RODs) and distractors with the orthogonal orientation as irrelevant-orientation distractors. We were able to examine the neural correlates of feature-based attention by varying whether the arrays contained RODs, under the assumption that RODs would receive differential processing compared with irrelevantorientation distractors because the RODs shared a feature with the target category.

An additional goal of this experiment was to determine whether the feature-based attention effects elicited by the presence of the RODs was independent of their spatial relationship to the target. As illustrated in Figure $1 a$ [for left visual field (LVF) targets], four conditions were tested: (1) RODs in the target visual field and irrelevant-orientation distractors in the nontarget visual field (target visual field condition, target-side ROD); (2) RODs in the nontarget visual field and irrelevant-orientation distractors in the target visual field (nontarget visual field condition, nontarget-side ROD); (3) RODs in both visual fields (both-sides ROD); a

\section{Relevant-orientation distractors on target side only (target-side ROD)}

\section{Relevant-orientation distractors on nontarget side only (nontarget-side ROD)}

\section{Relevant-orientation distractors on both sides} (both-sides ROD)

Relevant-orientation
distractors on neither
side (no ROD)
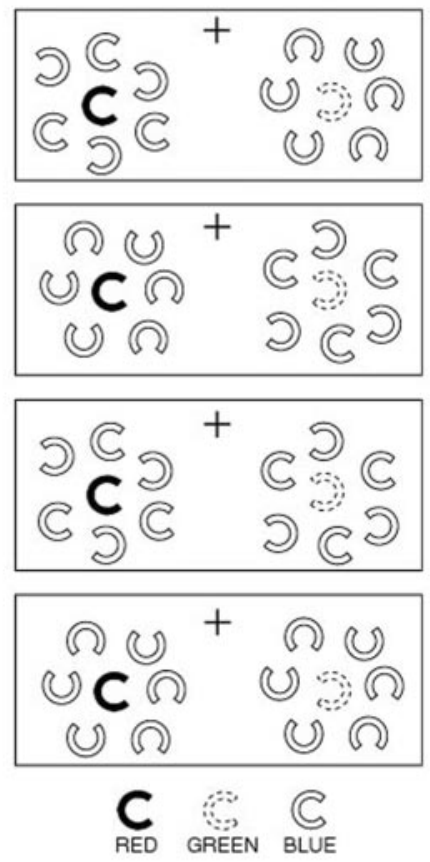

b

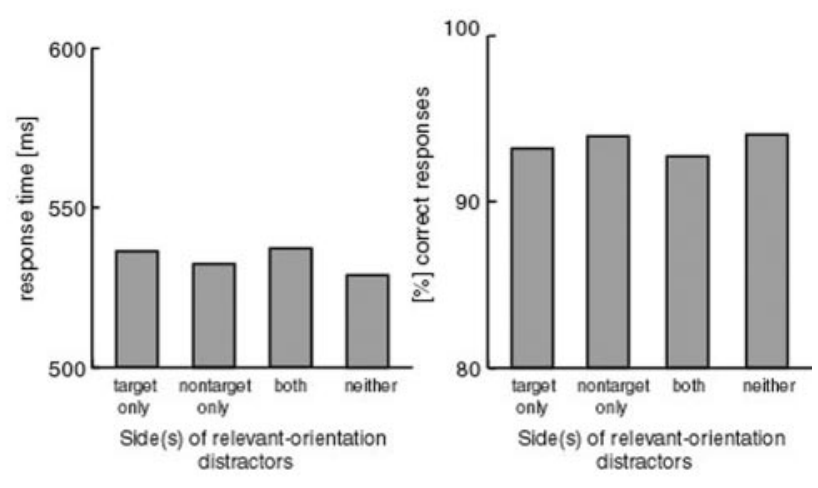

Figure 1. a, Stimulus arrays used in the first experiment. Stimuli consisted of arrays of C-shaped items placed to the left and right of fixation, with one distinctively colored item in each field (the red C in the LVF and the green ( in the RVF). One of these colors indicated the target for half of the trial blocks, and the other color indicated the target for the other half. Distracter items (blue (s) were placed in both visual fields surrounding the target and the potential target. The orientation of the distracters was either left-right like the target (ROD) or up- down (irrelevant-orientation distractors). The location of RODs and irrelevant-orientation distractors was varied to generate four different distractor distributions: (1) RODs in the target visual field and irrelevant-orientation distractors in the opposite field (target-side ROD); (2) RODs in the nontarget visual field and irrelevant-orientation distractors in the target field (nontarget-side ROD); (3) RODs in both visual fields (both-sides ROD); and (4) irrelevantorientation distractors in both visual fields with no RODs (no ROD). $b$, Behavioral results (experiment 1). The average response times (left) and percentage of correct responses (right) for the four experimental conditions are shown.

and (4) a control condition with irrelevant-orientation distractors in both visual fields (no ROD). Note, however, that although the distractors in a given cluster all had either a horizontally or vertically oriented gap, the exact position (left or right, top or bottom) varied randomly from distractor to distractor.

It should be emphasized that the location of the target item could always be identified solely by relying on its unique color. The distractors 
provided no information about target location or identity, and observers had no incentive to attend to them. Indeed, RODs could potentially lead to response-level interference, and this provided a disincentive for attending to them.

Stimuli were back-projected from a video graphics array-controlled video projector (SHARP XG-SVIE) onto a screen at a viewing distance of $120 \mathrm{~cm}$. The screen was gray $\left(8.0 \mathrm{~cd} / \mathrm{m}^{2}\right)$ and contained a permanently visible fixation point at the center of the display. Subjects were instructed to fixate this point and to minimize blinking. Fixation was monitored by an infrared video camera with a zoom lens; trials with eye movements were removed from all analyses. Stimulus arrays were presented for 700 $\mathrm{msec}$, with a variable duration blank interval of 1500-1600 msec between arrays. Subjects performed 10 trial blocks, 5 with red as the target color and 5 with green as the target color. Each block consisted of 160 stimulus presentations, with 40 presentations per condition (target-side ROD, nontarget-side ROD, both-sides ROD, and no ROD), yielding at total of 400 trials per subject for every condition.

Stimuli and procedure (experiment 2). Experiment 2 was designed to demonstrate that the effects observed in experiment 1 reflected the match or mismatch between the target and distractor features rather than the specific gap positions used in that experiment. The stimuli and procedure of experiment 2 were identical to those of experiment 1 , except that each target and distractor was rotated by $90^{\circ}$ (i.e., the targets had top or bottom gaps, RODs also had top or bottom gaps, and irrelevantorientation distractors had left or right gaps).

Stimuli and procedure (experiment 3; control experiment). In experiments 1 and 2, the orientation of the target gap matched the orientation of the surrounding distractor gaps in some conditions and mismatched them in other conditions. It is, therefore, possible that the effects observed in those experiments were caused by low-level stimulus differences rather than attentional processes. To assess this possibility, the stimulus configurations used in experiment 1 were presented in experiment 3 , but the subjects performed a fixation task in which these stimuli were irrelevant. If the effects observed in experiments 1 and 2 were attributable to low-level stimulus differences, then the same effects should be observed in this control experiment.

The stimuli and procedure were identical to those of experiment 1 , except that two small gray squares $\left(\right.$ size, $0.3 \times 0.3^{\circ}$ ) were presented $0.3^{\circ}$ above and below fixation (see Fig. $6 b$ ). The two squares were equal in brightness in $50 \%$ of the trials and differed in $50 \%$ of the trials. The subjects were instructed to press one button when the two squares matched in brightness (right index finger) and to press a different button when they did not match (right middle finger). Match and nonmatch trials were combined randomly with the four stimulus conditions of experiment 1 .

Subjects. All three experiments were undertaken with the understanding and written consent of the subjects. Subjects were paid for participation. Thirteen subjects (mean age, 26.5 years; 11 females) participated in the first experiment, 10 subjects (mean age, 23.0 years; 8 females) participated in the second experiment, and 10 subjects (mean age, 24.0 years; 7 females) participated in the third experiment. All subjects were righthanded, with normal color vision and normal or corrected-to-normal visual acuity.

Data recording. The magnetoencephalogram (MEG) and EEG signals were recorded simultaneously using a BTi Magnes 2500 whole-head MEG magnetometer system with 148 sensors (Biomagnetic Technologies) for the MEG and an electrode cap in conjunction with a 32-channel Synamps amplifier (NeuroScan) for the EEG. Electrode locations were chosen according to standard electrode montage of the American Electroencephalographic Society (1994) (Fpz, Fz, Cz, Pz, Oz, Iz, Fp1, Fp2, F7, F8, F3, F4, FC1, FC2, T7, T8, C3, C4, CP1, CP2, P7, P8, P3, P4, PO3, PO4, $\mathrm{PO} 7, \mathrm{PO} 8, \mathrm{IN} 3, \mathrm{IN} 4)$. The EEG was recorded with reference to the right mastoid. The MEG and EEG signals were filtered with a bandpass of DC $-50 \mathrm{~Hz}$ and digitized with a sampling rate of $254 \mathrm{~Hz}$. Artifact rejection was performed offline by removing epochs with peak-to-peak amplitudes exceeding a threshold of $3.0 \times 10^{-12} \mathrm{~T}$ for the MEG and $100 \mu \mathrm{V}$ for the EEG.

EEG electrode positions and the sensor frame coordinate system were spatially coregistered [i.e., the individual electrode setup together with anatomical landmarks (nasion and left and right preauricular points) were digitized] by using a 3Space Fastrak system (Polhemus). The locations of these landmarks in relation to sensor positions were derived on the basis of precise localization signals provided by five spatially distributed coils attached to the head with a fixed spatial relationship to the landmarks. The $32 \mathrm{EEG}$ electrode locations were also digitized to permit coregistration with the coordinate system. To compute the grand average activity over all subjects, the coordinate system for each subject was readjusted to the coordinate system of the Montréal Neurological Institute (MNI) brain (average of 152 T1-weighted stereotaxic volumes; see www.bic.mni.mcgill.ca/cgi/icbm_view/), the anatomical surface structure of which was segmented and used for the grand average source analyses.

ERMF source analysis was performed using the multimodal neuroimaging software Curry 4.0 (Philips Electronics) in the following way. First, on the basis of a high-resolution MR scan (neuro-optimized GE Signa LX $1.5 \mathrm{~T}$ scanner; T1-weighted three-dimensional spoiled gradient echo sequence; $256 \times 256$ matrix; field of view, $25 \times 25 \mathrm{~cm}$; 124 slices; slice thickness, $1.5 \mathrm{~mm}$; echo time, $6 \mathrm{msec}$; repetition time, $20 \mathrm{msec}$, flip angle, $30^{\circ}$ ), a three-dimensional reconstruction of the head, CSF space, and cortical surface was created for each subject using a boundary element model (BEM) (Hämäläinen and Sarvas, 1989; Fuchs et al., 1998). Second, a realistic volume conductor model (CSF-layer BEM with $1510 \pm 147$ nodes) was used in conjunction with the observed ERMF response to estimate a model of the distribution of current over the cortical surface by means of the minimum norm least squares method (L2 norm) (Hamalainen and Ilmoniemi, 1994; Fuchs et al., 1999). We will refer to these distributions as "source density estimates." In this model, the threedimensional grid of the cortical surface served as a predefined source compartment containing $13520 \pm 2430$ homogenously spaced elementary dipoles with no orientation constraint (rotating dipoles). Inherent in the minimum norm least squares model is the natural bias toward high gain source locations that would overemphasize superficial source locations. To compensate for this, we added an additional model term that weighs the estimated currents to account for the lower gains of deeper dipole components (Curry User Guide; Philips Electronics). Finally, to prevent over-fitting of the data, the regularization parameter that links the model term to the data were determined by the $\chi^{2}$ criterion, relying on the assumption that the data misfit is in the order of the amount of noise in the data. Noise was estimated from baseline magnetic activity within a period of $100 \mathrm{msec}$ preceding stimulus onset.

Statistical analysis. ERP and MEG waveforms were first collapsed over the two target colors (red and green) and then averaged separately for the four ROD distributions (target-side RODs, nontarget-side RODs, bothsides RODs, and no RODs) of the right and left visual field targets. Independent repeated-measures ANOVA (RANOVA) were then performed to validate ROD-related and target-related effects.

For statistical analysis of the ROD-related effects, mean amplitude measures in two time windows (140-190 and 200-290 msec) were subjected to separate three-way $(2 \times 2 \times 2)$ RANOVAs with the following factors: (1) electrode site (PO7, PO8); (2) presence or absence of RODs contralateral to the electrode site; and (3) presence or absence of RODs ipsilateral to the electrode site. The second factor was constructed by contrasting the conditions target-side ROD plus both-sides ROD with the conditions nontarget-side ROD plus no ROD (of targets in the visual field contralateral to the electrode). The levels of the third factor were derived by contrasting the conditions both-sides ROD plus nontargetside ROD with the conditions target-side ROD plus no ROD.

For statistical analysis of the target-related effects (N2pc effects), a four-way $(2 \times 2 \times 2 \times 2)$ RANOVA was performed on the mean amplitude between 200 and $300 \mathrm{msec}$ with the following factors: (1) electrode site (PO7, PO8); (2) target visual field (contralateral vs ipsilateral); (3) presence or absence of RODs on the target side; and (4) presence or absence of RODs on the nontarget side. The particular selection of time windows and electrode sites for the statistical analysis of ROD-related and target-related effects was guided by observations of previous studies showing maximum ERP effects of feature (color) discrimination at these electrode sites between 140-190 and 200-290 msec (Hopf et al. 2002b) 
and maximum effects of target lateralization (N2pc effects) between 200 and $300 \mathrm{msec}$ (Hopf et al., 2000, 2002a).

\section{Results \\ Experiment 1 \\ Behavioral data}

Figure $1 b$ shows average response times (left panel) and average percentages of correct responses (right panel) for the four different ROD distributions (data are collapsed over the target visual field). Responses tended to be slightly slower and less accurate when RODs were present on the side of the target (target-side ROD and both-sides ROD) compared with when they were absent (no ROD) or appeared in the nontarget visual field (nontarget-side ROD). A three-way RANOVA was conducted on the response time data with the factors target visual field (left, right), presence or absence of RODs on the target side, and presence or absence of RODs on the nontarget side. This analysis yielded a significant main effect of the presence or absence of RODs on the target side $\left(F_{(1,12)}=20.45 ; p<0.001\right)$ and a smaller, but significant, effect of the presence or absence of RODs on the nontarget side $\left(F_{(1,12)}=7.62 ; p<0.05\right)$ but no effect of target visual field $(F<1)$. No interactions were significant. A parallel analysis was conducted for the percentage of correct responses, and this yielded no significant main effects or interactions.

\section{ERP/ERMF waveforms and scalp distributions}

$R O D$-related responses. ERP responses at the P07 (left occipital) and the P08 (right occipital) electrode sites are shown for left visual field targets in Figure $2 a$ and for right visual field (RVF) targets in Figure $2 b$. In separate rows, waveforms from the three conditions in which RODs were present (target-side ROD, nontarget-side ROD, both-sides ROD; solid lines) are superimposed onto the waveform of the condition in which RODs were absent (no ROD; dashed lines). In each case, the amplitude in the N1 latency range was more negative when the contralateral visual field contained an ROD compared with the no ROD condition. We refer to this effect as the ROD-related negativity (Fig. $2 a$, unfilled arrows). The ROD-related negativity was observed over the left hemisphere when an ROD was in the right visual field (regardless of the visual field of the target), and it was observed over the right hemisphere when an ROD was in the left visual field (regardless of the visual field of the target). The ROD-related negativity was present in both hemispheres when both visual fields contained RODs. This pattern was also observed in the N2 latency range (marked with filled arrows).

The early effect was quantified as the mean amplitude between 140 and $190 \mathrm{msec}$, and the late effect was quantified as the mean amplitude between 200 and $290 \mathrm{msec}$; for both, the measurements were taken from the P07 and P08 electrode sites, using the $100 \mathrm{msec}$ prestimulus interval as a baseline. Separate RANOVAs were conducted for each time range with factors of electrode hemisphere (left or right), presence or absence of RODs contralateral to the electrode site, and presence or absence of RODs ipsilateral to the electrode site. For both the early and late phases of the ROD-related negativity, a significant effect was found for the presence of an ROD in the contralateral visual field (early phase: $F_{(1,12)}=31.9, p<0.0001$; late phase: $F_{(1,12)}=7.0, p<$ $0.05)$, but the presence of an ROD in the ipsilateral visual field did not have a significant effect (early phase: $F_{(1,12)}=1.88, p=0.19$; late phase: $F_{(1,12)}=0.94, p=0.35$ ). There was no significant interaction between electrode hemisphere and presence of an ROD in the contralateral visual field, but there was a trend in the early phase (early phase: $F_{(1,12)}=4.06, p=0.068$; late phase: $\left.F_{(1,12)}=0.43, p=0.52\right)$.
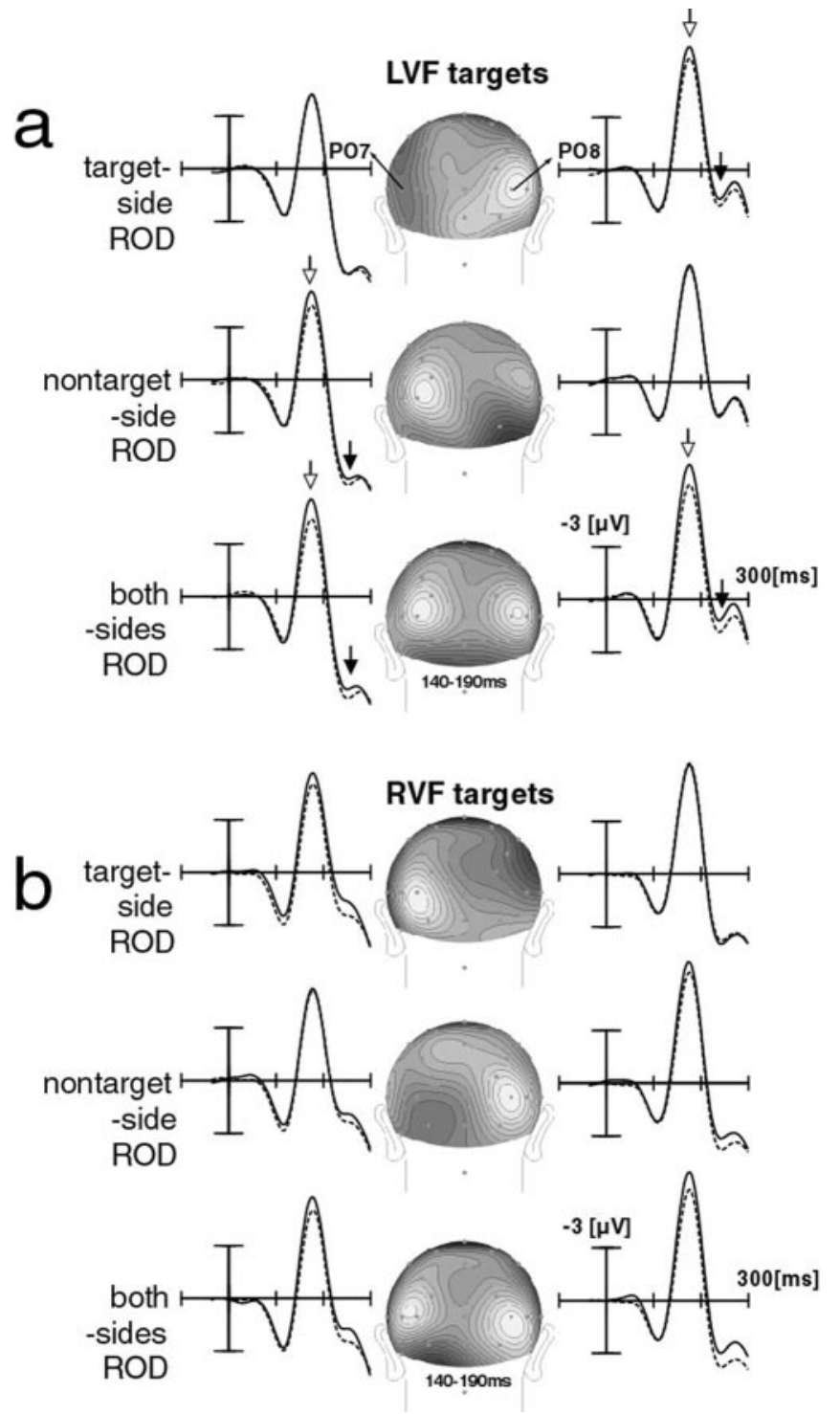

Figure 2. a, ROD-related effects for left visual field targets (experiment 1). ERP waveforms elicited by target-side ROD (top), nontarget-side ROD (middle), and both-sides ROD (bottom, solid lines) trials superimposed onto waveforms of the no ROD condition (dashed lines) at selected electrode sites from the left parieto-occipital (P07) and the right parieto-occipital (P08) scalp are shown. The ROD-related negativity is indicated by unfilled arrows (early portion) and filled arrows (later portion). The middle column illustrates the distribution of the RODrelated negativity (mean effect between 140 and $190 \mathrm{msec}$ ) by showing voltage difference maps target-side ROD minus no ROD (top), nontarget-side ROD minus no ROD (middle), and both-sides ROD minus no ROD (bottom). Positive to negative voltage differences are scaled from dark to bright, repectively. $b$, ROD-related effects for right visual field targets.

Figure $3 a$ shows a more detailed picture of the size and time course of the ROD-related negativity by plotting the difference between each of the three conditions with RODs and the condition with no RODs (target-side ROD minus no ROD, thin solid line; nontarget-side ROD minus no ROD, dash-dot line; bothsides ROD minus no ROD, bold solid line). The ROD-related negativity began at $\sim 140 \mathrm{msec}$ in all three conditions and extended until $\sim 300 \mathrm{msec}$, with peaks at $\sim 170$ and $270 \mathrm{msec}$. To analyze the onset latency of the ROD-related negativity in the three conditions, $t$ tests comparing no ROD with target-side ROD, nontarget-side ROD, and both-sides ROD were computed based on a short moving time window (three samples, $\sim 12 \mathrm{msec}$ ) centered at consecutive time samples between 100 and $200 \mathrm{msec}$. 


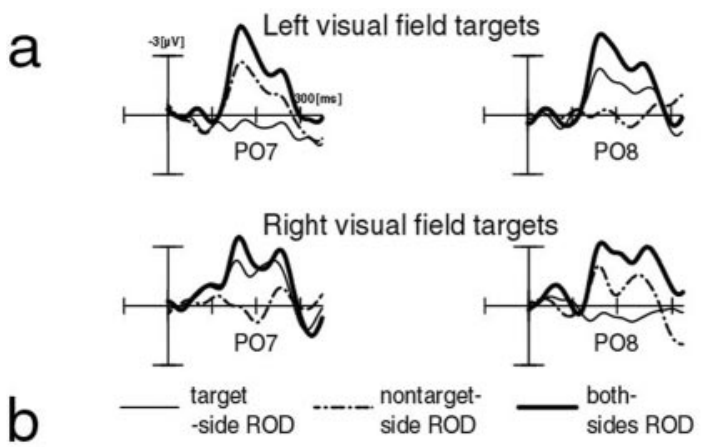

\section{RVF target}

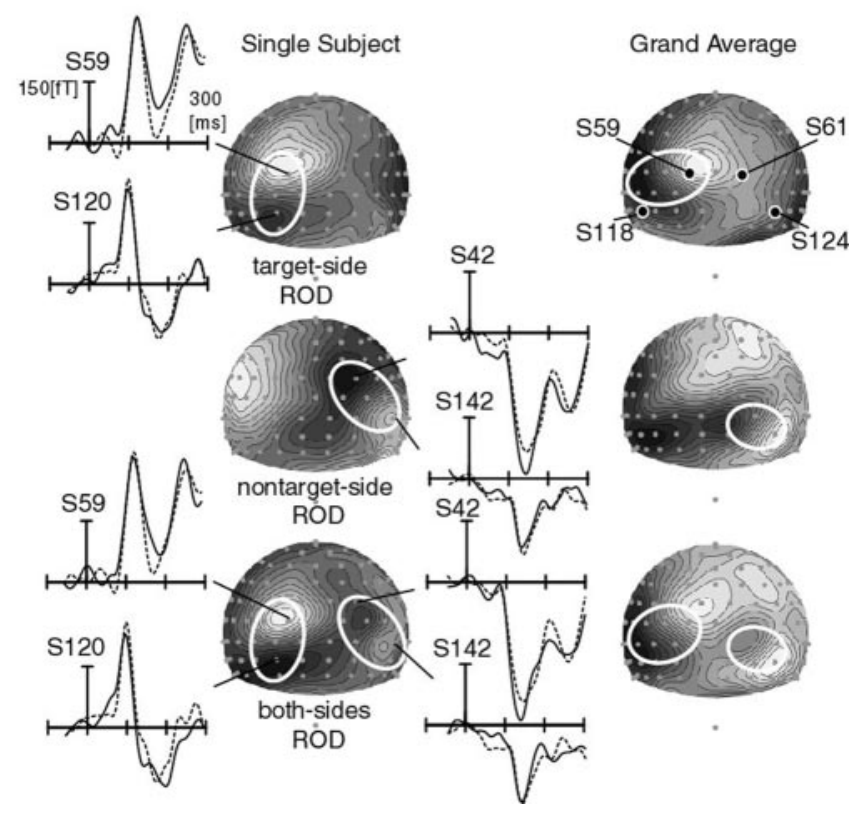

Figure 3. $a$, ERP difference waves target-side ROD minus no ROD (solid thin line), nontargetside ROD minus no ROD (dash-dot line), and both-sides ROD minus no ROD (solid thick line) for left visual field targets and right visual field targets at selected electrode sites. $b$, ERMF waveforms and distributions of one observer (left) and the grand average (right). ERMF waveforms are shown from selected sensor sites that flank the efflux-influx reversal of the magnetic flux field (white ellipses). Waveforms elicited by target-side ROD (solid lines; top left waveforms at S59 and S120), nontarget-side ROD (solid lines; middle right waveforms at S42 and S142), and both-sides ROD (solid lines at the bottom left and right) trials are superimposed onto waveforms of the no ROD condition (dashed lines). The four black dots in the top right topographical map (grand average) highlight the sensor sites from which measures were taken to validate the ROD-related effects in the magnetic waveforms.

These analyses were conducted on waveforms at electrodes contralateral to the RODs (collapsed across LVF and RVF targets). Significant voltage differences were present beginning in the 145$157 \mathrm{msec}$ time interval in all three conditions, which suggests that the onset latency does not depend on the location of the RODs with respect to the target.

Figure $3 b$ illustrates ERMF distributions (RVF targets only) of the difference waves (top, target-side ROD minus no ROD; middle, nontarget-side ROD minus no ROD; bottom, both-sides ROD minus no ROD). The left column shows the data for an individual observer, and the right column shows the grand average data. For the individual observer, MEG waveforms are shown from representative sensor sites over the left (S59, S120) and right (S42, S142) posterior hemisphere. In the field distribution maps, regions where the magnetic flux is leaving the head (an efflux) appear as dark areas, and regions where the magnetic flux is entering the head (an influx) appear as light areas. Regions showing magnetic efflux-influx reversal roughly correspond to the location of the underlying current (indicated by white ellipses in the maps). In the ERMF difference maps of the target-side ROD condition, a strong efflux-influx reversal is visible over the left posterior hemisphere in the grand average and in the individual observer (ellipses over the left hemisphere); conversely, for the nontarget-side ROD condition, an efflux-influx reversal appears over the right posterior region (ellipses over the right hemisphere). Consistent with the bilateral effect in the both-sides ROD condition, efflux-influx reversal regions are seen over both occipital hemispheres (ellipses over both hemisphere). A qualitatively similar ERMF distribution is observed for the grand average (see ellipses in the right side maps).

According to the "right-hand-rule," the efflux-influx region over the left occipital cortex corresponds to a current dipole with left and outward orientation, and the efflux-influx region over the right occipital cortex corresponds to a right and outward current dipole orientation, with both dipoles showing the same polarity. A comparison of the magnetic field distribution with that of the electrical data (Fig. 2b) confirms this interpretation. The ROD-related negativity shows the same polarity over the left and right scalp, and the ERP field maxima topographically correspond with the efflux-influx reversal of the ERMF of the region. Because the current origin corresponds with the polarity reversal of the magnetic flux field, the ROD-related negativity is best illustrated by showing responses from sensor sites that flank the efflux-influx reversal.

Figure $3 b$ (right) shows ERMF waveforms of an individual observer. The ROD-related effects are visible as the amplitude difference between waveforms that change relative polarity in the 140-200 msec time window (early and late phase). For example, in the top left waveforms in Figure $3 b$ taken from left hemisphere sensor sites, the solid line (target-side ROD) in sensor S59 shows an upward polarity relative to the dashed line (no ROD), whereas in sensor S120 the solid line shows a downward polarity relative to the dashed line. The same polarity reversal is seen in the right hemisphere at sensors S42 and S142. For statistical validation of the ROD-related effects in the magnetic data, a three-way RANOVA was computed for the 140-190 msec time range with factors of: (1) sensor site (left VF sensors, right VF sensors); (2) presence or absence of RODs contralateral to the sensor site; and (3) presence or absence of RODs ipsilateral to the sensor site. Measurements were taken from the sensor sites that are highlighted by the four black dots in the right top topographical map in Figure $3 b$ (grand average). To compensate for the field polarity inversion across sensor sites in each hemisphere, we inverted the values from sensor sites S118 and S61 and added them to the values from sites S59 and S124, respectively. As in the electrical data, a significant effect was found for the presence of an ROD in the contralateral visual field $\left(F_{(1,12)}=8.43 ; p<0.05\right)$, but the presence of an ROD in the ipsilateral visual field did not have a significant effect $\left(F_{(1,12)}<1\right)$. There was also no significant interaction between sensor hemisphere and presence of an ROD in the contralateral visual field $\left(F_{(1,12)}=1.39 ; p=0.26\right)$.

To summarize, the presence of a task-relevant feature (left or right gaps) in the distractors in a given visual field led to a change in the ERP/ERMF response between 140 and $-300 \mathrm{msec}$ (the ROD-related negativity). The ERP and ERMF scalp topography shows that this effect reflects the location of the RODs, independent of the location of the target item. The onset latency of the ROD-related negativity was also independent of target location. 


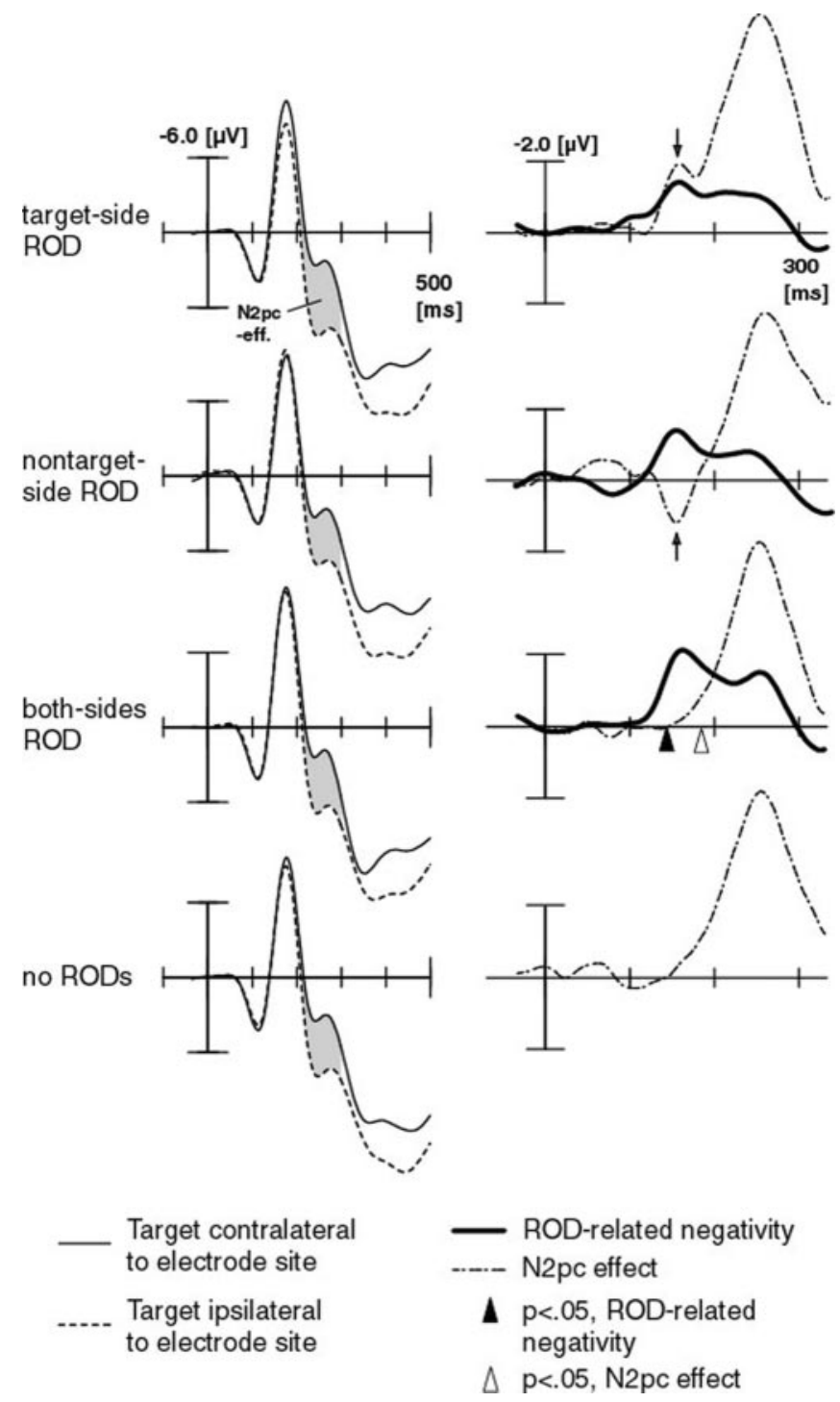

Figure 4. Target-related effects (N2pc effect). Left, Average waveforms elicited by target items contralateral (solid line) and ipsilateral (dashed line) to electrode sites P07/8. Data were collapsed over electrode sites to simplify presentation. The N2pc effect is highlighted by the gray areas between waveforms. Right, Time course of the N2pc effect shown as difference waves (contralateral targets minus ipsilateral targets; broken lines) and the ROD-related negativity, as in Figure $3 a$ (thick solid line). The arrows indicate that the ROD-related negativity is visible in the N2pc difference waves of the target-side ROD and nontarget-side ROD condition. Triangles mark the onset latency of a significant ROD-related effect (filled) and the N2pc effect (unfilled) of the both-sides ROD condition.

These observations, together with the fact that guidance by color (red, green) would have been sufficient to locate the target item, suggests that the ROD-related negativity reflects differential processing of the RODs that occurs solely because they possess a feature that was specified in the attentional set (i.e., the target template). Experiment 2, described below, will provide evidence that it is the match with the attentional set that produces the ROD-related negativity rather than the presence of a left or right gaps per se.

Target-related responses. Figure 4 (left) superimposes waveforms for contralateral versus ipsilateral target locations with respect to the hemisphere of the electrode site, displayed separately for all four conditions. This superimposition makes it possible to observe the target-related N2pc component. To simplify the presentation of waveforms, responses at electrode sites PO7 and PO8 were collapsed to create average waveforms for attended targets in the visual field contralateral (solid black traces) and ipsilateral (dash-dot traces) to the electrode site. The N2pc component can be observed as a more negative-going voltage for contralateral relative to ipsilateral targets (gray region). A large N2pc effect was observed in all four conditions in the typical time range, between $\sim 180$ and 300 msec. For statistical validation, a four-way repeated measures RANOVA with the factors electrode hemisphere (left vs right), target visual field (contralateral vs ipsilateral), presence or absence of RODs on the target side, and presence or absence of RODs on the nontarget side was performed on the mean voltage measured between 200 and $300 \mathrm{msec}$. In this analysis, a significant main effect of target visual field was observed $\left(F_{(1,12)}=41.35 ; p<0.0001\right)$ which validates the presence of a prominent $\mathrm{N} 2$ pc effect. In addition, we observed significant twoway interactions of target visual field with the presence or absence of RODs in the target visual field $\left(F_{(1,12)}=43.43 ; p<0.0001\right)$ and the nontarget visual field $\left(F_{(1,12)}=42.76 ; p<0.0001\right)$. Both interactions reflect the fact that the N2pc effect overlaps with the late phase of the ROD-related negativity in the target-side ROD and nontarget-side ROD condition, which is also lateralized. This, unfortunately, makes it impossible to directly compare the size of the N2pc component across conditions (see below).

Figure 4 (right) illustrates the relative time course of the N2pc effect and the ROD-related negativity separately for each condition. Shown are left visual field minus right visual field target difference waveforms, which represent the N2pc component elicited by the target (broken traces), superimposed onto difference waveforms of the corresponding ROD-related negativities (solid traces). Note that the solid traces show the same difference waves as Figure 3a, except that responses from electrodes contralateral to the ROD location were collapsed across left visual field and right visual field target trials. As mentioned above, the N2pc difference waves still show the ROD-related effect in the target-side ROD condition and with reversed polarity (because of the direction of subtraction) in the nontarget-side ROD condition (arrows). Because waveforms are compared between visual fields, the ROD-related effect is cancelled in the N2pc difference waveform of the both-sides ROD condition, in which a bilateral effect was obtained. Hence, a reasonable comparison of the onset latencies of the ROD-related negativity and the N2pc effect was only possible for the both-sides ROD condition. The earliest significant difference was obtained in the 185-197 msec time window (moving-window $t$ tests between 150 and $250 \mathrm{msec}$ ), $30 \mathrm{msec}$ after the onset of the ROD-related negativity (see arrowheads at abscissa). The N2pc is known to reflect the operation of focusing attention onto the target in multi-item displays (Luck and Hillyard, 1994a; Luck et al., 1997a). Hence, the earlier onset (30 $\mathrm{msec}$ ) of the ROD-related negativity shows that the neural operation underlying the registration of searched-for feature values does indeed precede the effects of focusing attention onto the target item. Nevertheless, there is a considerable period of overlap (200-300 msec) between these processes.

\section{ERMF current source localization}

$R O D$ related effects. The $\mathrm{ROD}-$ related negativity observed in the ERP recordings was accompanied by ERMF effects in the same time range. The main purpose of the ERMF recordings was to localize more precisely the neural generator source of this effect. To minimize ERMF activity that was not related to the RODrelated negativity, source density estimates were computed from ERMF difference waves in which the trials without RODs (no ROD trials) were subtracted from each of the other trial types 
a
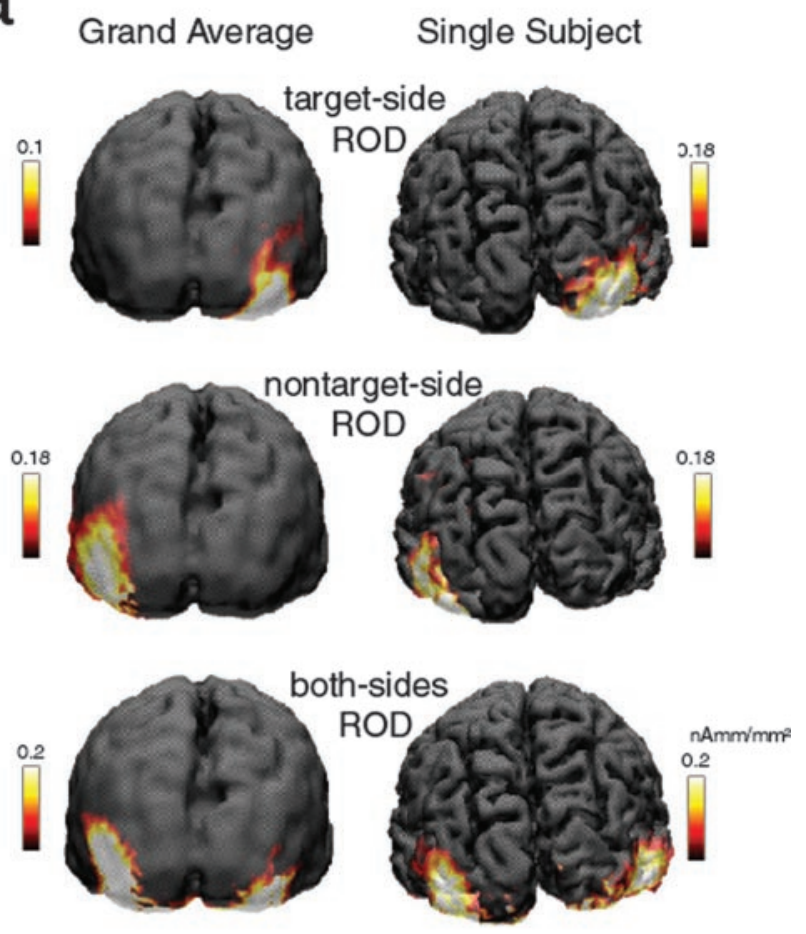

LVF target

b

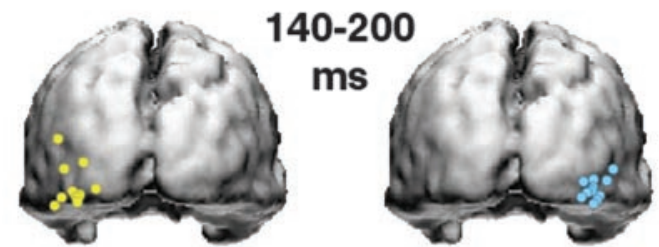

target-side ROD minus no ROD

$O$ target in RVF

target in LVF

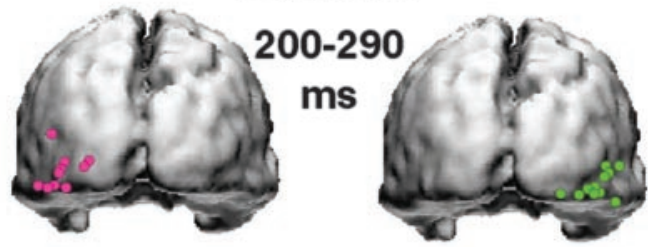

target-side ROD minus no ROD

target in RVF

target in LVF

Figure 5. Source density estimates (ERMF data of experiment 1). a, Source density estimates for the ROD-related negativity (140-200 msec) of the grand average (left) and one single subject (right). Shown are results for left visual field target trials only. $b$, Maxima of the source density estimate for the ROD-related negativity of individual subjects (each dot represents one subject). Maxima were computed in early (140-200 msec; yellow and blue dots) and late (200-290 msec; pink and green dots) time ranges, using individual anatomical data, and then transformed into the MNI reference space.

(target-side ROD, nontarget-side ROD, and both-sides ROD). Figure $5 a$ shows the source density estimates in the N1 time range (140-190 msec) of the grand average and of one single subject for left visual field targets (similar but complementary effects were target-side

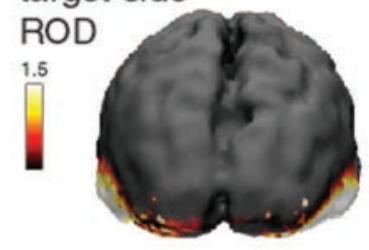

nontarget-side

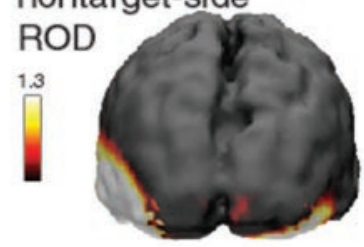

both-sides

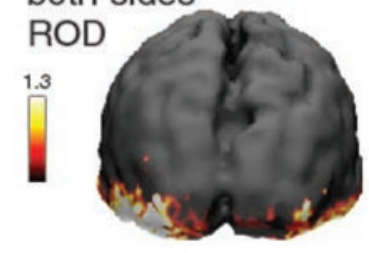

N2pc (tROD)

LVF-target minus

RVF-target

ROD-related negativity

(target-side ROD)

- target in RVF

- target in LVF

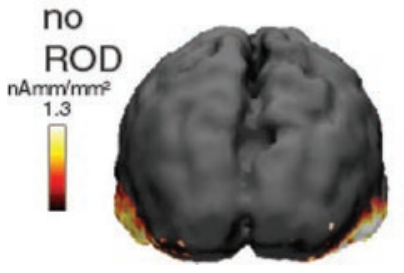

Figure 6. Source density estimates of the N2pc effect. Left, Source density estimates of the grand average N2pc for the target-side ROD, nontarget-side ROD, and both-sides ROD condition. Right, Source density maxima of the N2pc of individual subjects (yellow dots) plotted on lateral hemisphere views together with the maxima of the ROD-related negativity (target-side ROD) between 140 and $200 \mathrm{msec}$ (red and blue dots), already shown in Figure $5 b$.

observed for right visual field targets). The underlying source activity originates from ventral occipito-temporal regions contralateral to the location of the RODs. Hence, in line with the ERP scalp topography, the hemispheric lateralization of the current source activity reflects the distribution of RODs and not that of the target item.

To compare the early and late phases of the ROD-related negativity, source density estimates were computed separately for the early $(140-190 \mathrm{msec})$ and late $(200-290 \mathrm{msec})$ time periods. Figure $5 b$ shows the location of the maximal source density estimate for the target-side ROD minus no ROD difference in individual subjects for the early and late time ranges. Source density maxima were computed for 11 subjects (for which a sufficient signal to noise ratio $>5$ allowed reliable estimates) using their individual anatomical data and then transformed into the MNI reference frame for illustration (see Materials and Methods). The distribution of source density maxima for the early and late time ranges showed almost perfect overlap, suggesting that they arise from similar, overlapping regions of cortex.

The N2pc effect. Source density estimates were also computed for the N2pc effect (200-300 msec) using LVF target minus RVF target difference waves, thus subtracting away any ERMF activity that was not lateralized with respect to the target (for a detailed justification of this approach, see Hopf et al., 2000). Figure 6 (left) shows the resulting source density estimate for the target-side ROD, nontarget-side ROD, both-sides ROD, and no ROD con- 
Table 1. Talairach coordinates of ERMF source density maxima (experiment 1)

\begin{tabular}{|c|c|c|c|c|c|c|c|}
\hline & \multirow[b]{2}{*}{ ROD at } & \multicolumn{3}{|c|}{ Left hemisphere } & \multicolumn{3}{|c|}{ Right hemisphere } \\
\hline & & $x$ & $y$ & $z$ & $x$ & $y$ & $Z$ \\
\hline \multicolumn{8}{|l|}{ ROD-related negativity } \\
\hline & Target side & 41.5 & -84.8 & -1.7 & -40.3 & -88.5 & -1.6 \\
\hline & Nontarget side & 44.6 & -80.0 & -2.0 & -40.8 & -87.2 & -5.1 \\
\hline & Both sides & 43.1 & -82.4 & -3.4 & -42.8 & -85.6 & -5.7 \\
\hline \multicolumn{8}{|l|}{ N2pc effect } \\
\hline & Target side & 54.7 & -61.4 & -6.4 & -56.8 & -62.0 & -6.3 \\
\hline & Nontarget side & 52.9 & -63.7 & -8.5 & -55.9 & -62.9 & -8.2 \\
\hline & Both sides & 51.6 & -66.0 & -5.5 & -55.0 & -65.8 & -8.3 \\
\hline & No & 50.6 & -67.3 & -6.8 & -54.9 & -68.0 & -4.5 \\
\hline
\end{tabular}

ditions. In all four conditions, the current sources underlying the $\mathrm{N} 2 \mathrm{pc}$ effect arose from ventral occipito-temporal areas of the left and right hemisphere, which replicates our previous N2pc localizations (Hopf et al., 2000, 2002a). The right side of Figure 6 shows the maxima of the N2pc effect (yellow dots) for 11 individual subjects together with the maxima of the early portion of the ROD-related negativity (red and blue dots). Although both the ROD-related negativity and the N2pc effect originate from the ventral occipito-temporal cortex, the maximum of the ROD-related negativity is clearly more posterior than the maximum of the N2pc effect. Table 1 summarizes the locations of the maxima (averaged across observers) of the RODrelated negativity and the N2pc effect for each experimental condition in the Talairach reference space (Talairach and Tournoux, 1988).

\section{Experiment 2}

Experiment 2 tests a possible alternative explanation for the results of experiment 1 . Specifically, the RODs always had a left or right gap, whereas the irrelevant-orientation distractors always had a top or bottom gap, and it is possible, although unlikely, that the ROD-related negativity reflects this low-level stimulus property rather than the match between the features of the RODs and the target. To test this, we simply rotated each target and distractor item by $90^{\circ}$. The pattern of results replicated the pattern that was observed in experiment 1 . RANOVAs were performed as in experiment 1. Again, a significant effect was found in the early $(140-190 \mathrm{msec})$ and the late $(200-290 \mathrm{msec})$ phases of the response for the factor presence or absence of an ROD in the contralateral visual field (early phase: $F_{(1,9)}=7.89, p<0.05$; late phase: $\left.F_{(1,9)}=38.49, p<0.0001\right)$. The presence or absence of an ROD in the ipsilateral visual field did not have a significant effect (early phase: $F_{(1,9)}=3.32, p=0.10$; late phase: $F_{(1,9)}=0.05, p=$ $0.85)$. No significant interactions with electrode hemisphere were observed. Hence, RODs again elicited a negativity over contralateral occipito-temporal scalp sites, although they had top and bottom gaps rather than left and right gaps. This is illustrated in Figure $7 a$. Thus, the ROD-related negativity reflects the match between the features of the distractors and the features of the target rather than the specific orientation of the gaps.

\section{Experiment 3}

Experiment 3 tested the possibility that the ROD-related negativity reflects the low-level physical mismatch between the target and the surrounding distractors. That is, the stimulus configurations in the target-side ROD and the both-sides ROD conditions were physically different from those in the nontarget-side ROD and no ROD conditions, and it is possible, although unlikely, that the ROD-related negativity reflects this low-level difference. This was tested by presenting the same stimuli used in experiment 1 but making them task irrelevant; subjects performed a brightness task at fixation instead. Brightness discrimination was used because it does not give rise to an attentional template that would match the set of features relevant for the peripheral items. Furthermore, because attention was closely focused around fixation, the peripheral items were also not passively attended. Figure $7 b$ shows very clearly that no ROD-related negativities were present in this experiment. The data were measured and analyzed just as in experiment 1 , and the effects of ROD presence or absence at contralateral scalp sites did not approach significance [early phase $(140-190 \mathrm{msec}): F_{(1,9)}=1.55, p=0.24$; late phase $(200-$ $\left.290 \mathrm{msec}): F_{(1,9)}=0.84, p=0.38\right)$. Thus, low-level stimulus confounds cannot explain the results of experiment 1 .

\section{Discussion}

The results of this study can be summarized as follows. When the distractors in a given visual field contained a feature that matched the target specification (a gap in the appropriate position), the ERP waveform became more negative between 140 and $300 \mathrm{msec}$ poststimulus (the ROD-related negativity). ERP scalp topography as well as current source localization based on simultaneously recorded ERMF data revealed that this modulation occurred primarily (or even entirely) within the hemisphere contralateral to the matching distractors, regardless of which side the target was on. Thus, the spatial locations of the matching features was represented at least coarsely. Control experiments demonstrated that this effect reflected the match between the distractor features and the target template rather than low-level stimulus confounds. The neural response reflecting the focusing of attention onto the location of the target (N2pc) began $\sim 30$ msec after the ROD-related negativity, although both processes showed considerable temporal overlap later on (200-300 msec). Current source localization revealed that the spatial peak of feature-related activity (the ROD-related negativity) was more posterior than the spatial peak of target location-related activity (the N2pc component).

The ROD-related negativity is analogous to single-unit effects observed in monkeys in which feature-specific neuronal activity depended on the presence of an attended feature, independent of where attention is focused (Motter, 1994; Treue and Martinez Trujillo, 1999). For example, Treue and Martinez-Trujillo (1999) reported that in macaque $\mathrm{MT}$, the firing rates of motion direction-selective cells were modulated to the extent that their direction selectivity matched the attended motion direction. Importantly, this effect was observed in MT neurons with receptive fields (RFs) away from the spatial focus of attention. Neural activity depended on the similarity between the attended feature value and the selectivity of the cell for it. Analogous effects have 
a

LVF target RVF target
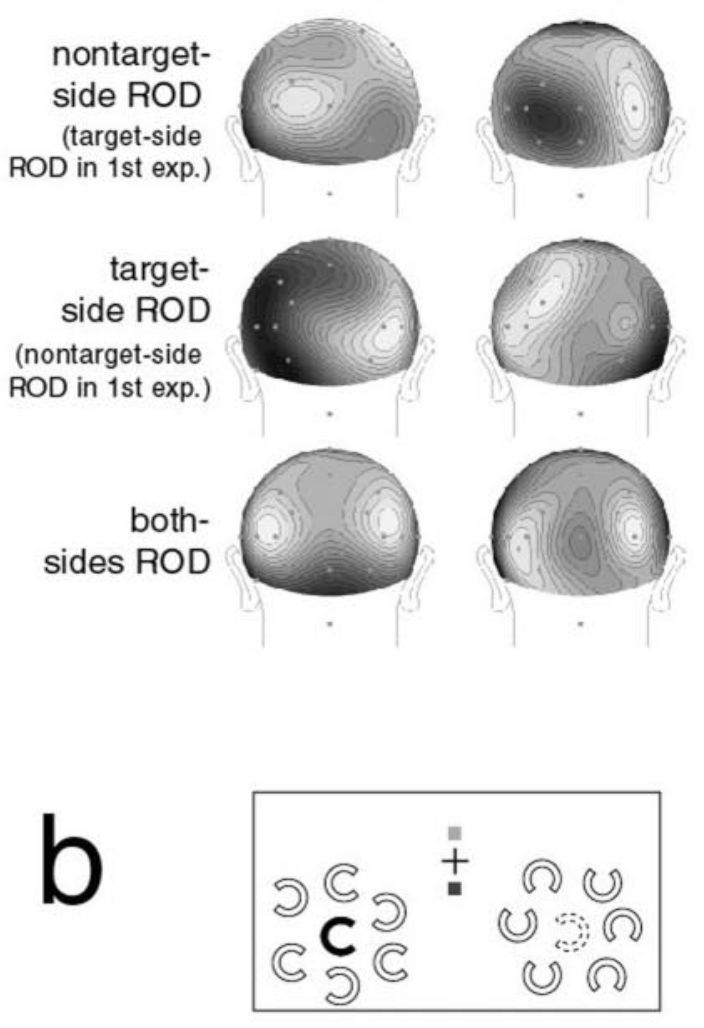

LVF target
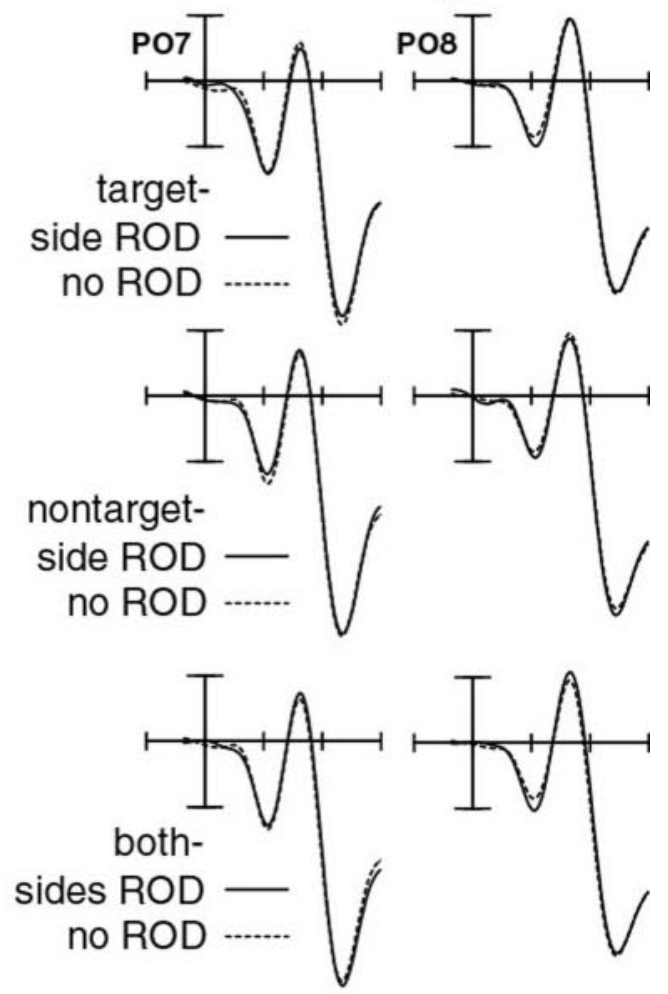

Figure 7. $a$, ROD-related negativity (experiment 2). Voltage difference maps nontarget-side ROD minus no ROD (top), target-side ROD minus no ROD (middle), and both-sides ROD minus no ROD (bottom). Positive to negative voltage differences are scaled from dark to bright, respec- been observed at a more macroscopic level in humans in an fMRI study (Saenz et al., 2002).

Likewise, Motter (1994) observed differential activity in color-responsive V4 neurons with RFs outside the spatial focus of attention, depending on their match with a color pre-cue. He suggested that such location-independent effects of feature selection may serve to highlight potential target locations in the visual field and may provide the neural basis for the parallelism in simple feature search. The earliest effects of feature-based attentional selection in the study by Motter (1994) appeared $\sim 150$ msec after stimulus onset and lasted, on average, for another $150 \mathrm{msec}$, consistent with the onset latency and duration of the ROD-related negativity observed here.

Despite these parallels, it is difficult to be certain whether the ERP/ERMF results observed in the present study reflect the same neural processes observed in these single-unit and fMRI studies (Luck, 1999). However, even if the ERP/ERMF effects do not reflect exactly the same neural activity, they certainly reflect the same type of process: a location-independent feature selection process that depends on the match between a given feature and an attentional template for the target.

It is instructive to compare the present results with previously reported feature-selective modulations of the ERP response in the N1 time range and beyond (Harter and Previc, 1978; Harter and Guido, 1980; Hillyard and Münte, 1984; Wijers et al., 1989; Anllo-Vento and Hillyard, 1996; Heslenfeld et al., 1997). In most of these studies, all stimuli were presented at attended locations, making it impossible to determine the relationship between attention to spatial and nonspatial features. Some studies, however, used a paradigm in which attention was sustained at a given location for a period of minutes, during which objects containing attended or unattended features were presented at the attended or ignored locations (Hillyard and Münte, 1984; Anllo-Vento and Hillyard, 1996). In these studies, attended features and unattended features yielded different ERP responses, just as in the present study, but these differences were observed only at the attended location (i.e., the match between the color or direction of motion of an object and the target template led to little or no differential ERP location when the object was presented at an ignored location). This led to the conclusion that the analysis of the nonspatial features of an object was contingent on the prior analysis of the location of the object. In contrast, the ROD-related negativity observed in the present study was completely independent of the location of the target.

These contrasting patterns of results can be explained quite easily by considering the nature of the tasks used in these experiments. In search tasks, like in the present study, target identification must be accomplished without preknowledge of the location of the target. Hence, search has to rely, at least initially, on some form of feature-based information about the target. It is, therefore, reasonable to expect that feature-based effects will occur before the selection of the target location. Indeed, we observed prominent ROD-related effects in the attended and the unattended visual field as the earliest correlates of attentional selection. The important finding of the present study is that this initial feature-based selection is feature value specific and pro-

$\leftarrow$

tively. The order of conditions was changed (top and middle) to allow a direct comparison with corresponding topographical maps in Figure 2, $a$ and $b . b$, ERP waveforms at the P07 and P08 electrode sites from experiment 3. Shown are waveforms that correspond to the conditions of target-side ROD (top), nontarget-side ROD (middle), and both-sides ROD (bottom; solid lines) trials from the first experiment, as shown in Figure $2 a$. 
vides a neural representation of the spatial distribution of the searched-for feature values independent of the distribution of other target-defining features (e.g., target color). Behavioral studies have provided related evidence, suggesting that featurebased effects mediate the attentive selection of even noncontiguous locations (Bichot et al., 1999; Cave, 1999) and that early feature selection immediately provides location information at least in a coarse manner (Cohen and Ivry, 1989).

It is important to emphasize that the target item in the present study was defined unambiguously by a unique and highly discriminable color, and it should not have been necessary to process information about gap location to find the target. This suggests that when it is necessary for the visual system to rely on feature-based selection, the registration of locations with any feature value that matches the attentional template is obligatory and automatic and cannot be blocked as with location precuing (Hillyard and Münte, 1984). A number of behavioral and electrophysiological findings in the literature are in line with this conclusion. For example, it has been shown that items at nontarget locations attract attention in visual search to the extent that they bear features of the searched-for set (Kim and Cave, 1995; Shui-I and Sperling, 1996; Theeuwes and Burger, 1998; Woodman and Luck, 1999; Arnott et al., 2001; Theeuwes et al., 2001). In particular, Folk and colleagues (Folk et al., 1992, 1994, 2002; Folk and Remington, 1998) and others (Yantis and Egeth, 1999) have shown that distractors matching the attentional set are highly effective at capturing attention when attention is not already strongly focused on a different location.

Theories of visual search have proposed that feature-based selection processes can guide the allocation of spatial attention to potential target objects (Wolfe et al., 1989; Treisman and Sato, 1990; Wolfe, 1994; Cave, 1999). In their revised feature integration model, Treisman and Sato (1990) suggest that attention controls locations in the master map via links to the feature maps before the actual operation of spatial attention comes into play (when necessary). Similar proposals are made by the guided search model of Wolfe et al. (1989) and the feature gate model of Cave (1999). Although, the issue of guidance was not directly addressed in the present study, the properties of the ROD-related negativity qualify it as a possible correlate of such a preattentional feature map that may guide attentional focusing. The RODrelated negativity reflects obligatory registration of attended feature values in any location of space, and it starts $\sim 30$ msec before (and overlaps with) the operation of focusing attention onto the target item (reflected by the N2pc effect). Furthermore, although both the N2pc and the ROD-related negativity arise from ventral occipito-temporal areas, the ROD-related negativity tended to have a more posterior distribution. This suggests an origin from a lower level in the visual processing hierarchy. It is, therefore, possible that the results of feature-based location selection in an intermediate extrastriate area (e.g., area V4) are fed forward directly to a higher region (e.g., inferotemporal cortex) where the target selection takes place. Additional experiments will be necessary to demonstrate whether the ROD-related negativity actually reflects a feature distribution map that serves to guide attentional focusing.

\section{References}

American Electroencephalographic Society (1994) Guideline seven: a proposal for standard montages to be used in clinical EEG. American Electroencephalographic Society. J Clin Neurophysiol 11:30-36.

Anllo-Vento L, Hillyard SA (1996) Selective attention to the color and direction of moving stimuli: electrophysiological correlates of hierarchical feature selection. Percept Psychophys 58:191-206.
Arnott SR, Pratt J, Shore DI, Alain C (2001) Attentional set modulates visual areas: an event-related potential study of attentional capture. Brain Res Cogn Brain Res 12:383-395.

Bichot NP, Cave KR, Pashler H (1999) Visual selection mediated by location: feature-based selection of noncontiguous locations. Percept Psychophys 61:403-423.

Brefczynski JA, DeYoe EA (1999) A physiological correlate of the 'spotlight' of visual attention. Nat Neurosci 2:370-374.

Cave KR (1999) The FeatureGate model of visual selection. Psychol Res 62:182-194.

Chelazzi L, Miller EK, Duncan J, Desimone R (1993) A neural basis for visual search in inferior temporal cortex. Nature 363:345-347.

Chelazzi L, Miller EK, Duncan J, Desimone R (2001) Responses of neurons in macaque area V4 during memory-guided visual search. Cereb Cortex 11:761-772.

Cohen A, Ivry R (1989) Illusory conjunctions inside and outside the focus of attention. J Exp Psychol Hum Percept Perform 15:650-663.

Connor CE, Preddy DC, Gallant JL, Van Essen DC (1997) Spatial attention effects in macaque area V4. J Neurosci 19:3201-3214.

Corbetta M, Miezin FM, Dobmeyer S, Shulman GL, Petersen SE (1990) Attentional modulation of neurall processing of shape, color, and velocity in humans. Science 248:1556-1559.

Folk CL, Remington R (1998) Selectivity in distraction by irrelevant featural singletons: evidence for two forms of attentional capture. J Exp Psychol Hum Percept Perform 24:847-858.

Folk CL, Remington RW, Johnston JC (1992) Involuntary covert orienting is contingent on attentional control settings. J Exp Psychol Hum Percept Perform 18:1030-1044.

Folk CL, Remington RW, Wright JH (1994) The structure of attentional control: contingent attentional capture by apparent motion, abrupt onset, and color. J Exp Psychol Hum Percept Perform 20:317-329.

Folk CL, Leber AB, Egeth HE (2002) Made you blink! Contingent attentional capture produces a spatial blink. Percept Psychophys 64:741-753.

Fuchs M, Drenckhahn R, Wischmann HA, Wagner M (1998) An improved boundary element method for realistic volume-conductor modeling. IEEE Trans Biomed Eng 45:980-997.

Fuchs M, Wagner M, Kohler T, Wischmann HA (1999) Linear and nonlinear current density reconstructions. J Clin Neurophysiol 16:267-295.

Hämäläinen MS, Ilmoniemi RJ (1994) Interpreting magnetic fields of the brain: minimum norm estimates. Med Biol Eng Comput 32:35-42.

Hämäläinen MS, Sarvas J (1989) Realistic conductivity geometry model of the human head for intrerpretation of neuromagnetic data. IEEE Trans Biomed Eng 36:165-171.

Harter MR, Guido W (1980) Attention to pattern orientation: negative cortical potentials, reaction time, and the selection process. Electroencephal Clin Neurophysiol 49:461-475.

Harter MR, Previc FH (1978) Size-specific information channels and selective attentionn: visual evoked potential and behavioral measures. Electroencephal Clin Neurophysiol 45:628-640.

Heinze HJ, Mangun GR, Burchert W, Hinrichs H, Scholz M, Münte TF, Gös A, Scherg M, Johannes S, Hundeshagen H, Gazzaniga MS, Hillyard SA (1994) Combined spatial and temporal imaging of brain activity during visual selective attention in humans. Nature 372:543-546.

Heslenfeld DJ, Kenemans JL, Kok A, Molenaar PCM (1997) Feature processing and attention in the human visual system: an overview. Biol Psychol 45:183-215.

Hillyard SA, Münte TF (1984) Selective attention to color and location: an analysis with event-related brain potentials. Percept Psychophys 36:185-198.

Hopf JM, Luck SJ, Girelli M, Hagner T, Mangun GR, Scheich H, Heinze H-J (2000) Neural sources of focused attention in visual search. Cereb Cortex 10:1233-1241.

Hopf JM, Boelmans K, Schoenfeld AM, Heinze H-J, Luck SJ (2002a) How does attention attenuate target-distractor interference in vision? Evidence from magnetoencephalographic recordings. Cognit Brain Res 15:17-29.

Hopf JM, Vogel E, Woodman G, Heinze HJ, Luck SJ (2002b) Localizing visual discrimination processes in time and space. J Neurophysiol 88:2088-2095.

Kim MS, Cave KR (1995) Spatial attention in visual search for features and feature conjunctions. Psychol Sci 6:376-380.

Kim MS, Cave KR (2001) Perceptual grouping via spatial selection in a focused-attention task. Vision Res 41:611-624. 
Luck SJ, Hillyard SA (1994a) Electrophysiological correlates of feature analysis during visual search. Psychophysiology 31:291-308.

Luck SJ, Hillyard SA (1994b) Spatial filtering during visual search: evidence from human electrophysiology. J Exp Psychol Hum Percept Perform 20:1000-1014.

Luck SJ, Girelli M, McDermott MT, Ford MA (1997a) Bridging the gap between monkey neurophysiology and human perception: an ambiguity resolution theory of visual selective attention. Cogn Psychol 33:64-87.

Luck SJ, Chelazzi L, Hillyard SA, Desimone R (1997b) Mechanisms of spatial selective attention in areas $\mathrm{V} 1, \mathrm{~V} 2$, and $\mathrm{V} 4$ of macaque visual cortex. J Neurophysiol 77:24-42.

Luck SJ (1999) Direct and indirect integration of event-related potentials, functional magnetic resonance images, and single-unit recordings. Hum Brain Mapp 8:115-120.

Moran J, Desimone R (1985) Selective attention gates visual processing in the extrastriate cortex. Science 229:782-784.

Motter B (1993) Focal attention produces spatially selective processing in visual cortical areas V1, V2, and V4 in the presence of competing stimuli. J Neurophysiol 70:909-919.

Motter BC (1994) Neural correlates of attentive selection for color or luminance in extrastriate area V4. J Neurosci 14:2178-2189.

O’Craven KM, Rosen BR, Kwong KK, Treisman A, Savoy RL (1997) Voluntary attention modulates fMRI activity in human MT-MST. Neuron 18:591-598.

Posner MI (1980) Orienting of attention. Q J Exp Psychol 32:3-25.

Saenz M, Buracas GT, Boynton GM (2002) Global effects of feature-based attention in human visual cortex. Nat Neurosci 5:631-632.

Shui-I S, Sperling G (1996) Is there feature-based attentional selection in visual search? J Exp Psychol Hum Percept Perform 22:758-779.
Talairach J, Tournoux P (1988) Co-planar stereotaxic atlas of the human brain: 3-dimensional proportional system: an approach to cerebral imaging. Stuttgard: Thieme Verlag.

Theeuwes J, Burger R (1998) Attentional control during visual search: the effect of irrelevant singletons. J Exp Psychol Hum Percept Perform 24:1342-1353.

Theeuwes J, Kramer AF, Atchley P (2001) Spatial attention in early vision. Acta Psychol (Amst) 108:1-20.

Treisman A, Gelade G (1980) A feature-integration theory of attention. Cogn Psychol 12:97-136.

Treisman A, Sato S (1990) Conjunction search revisited. J Exp Psychol Hum Percept Perform 16:459-478.

Treue S, Martinez Trujillo JC (1999) Feature-based attention influences motion processing gain in macaque visual cortex. Nature 399:575-579.

Wijers AA, Mulder G, Okita T, Mulder LJM, Scheffers MK (1989) Attention to color: an analysis of selection, controlled search, and motor activation, using event-related potentials. Psychophysiology 26:89-109.

Wolfe J (1994) Guided search 2.0 A revised model of visual search. Psychonomic Bull Rev 1:202-238.

Wolfe JM, Cave KR, Franzel SL (1989) Guided search: an alternative to the feature integration model for visual search. J Exp Psychol Hum Percept Perform 15:419-433.

Woodman GF, Luck SJ (1999) Electrophysiological measurement of rapid shifts of attention during visual search. Nature 400:867-869.

Yantis S, Egeth HE (1999) On the distinction between visual salience and stimulus-driven attentional capture. J Exp Psychol Hum Percept Perform 25:661-676. 